\title{
Lag effect of climatic variables on dengue burden in India
}

\section{Original Paper}

Cite this article: Kakarla SG, Caminade C, Mutheneni SR, Morse AP, Upadhyayula SM, Kadiri MR, Kumaraswamy S (2019). Lag effect of climatic variables on dengue burden in India. Epidemiology and Infection 147, e170, 1-10. https://doi.org/10.1017/ S0950268819000608

Received: 3 July 2018

Revised: 1 September 2018

Accepted: 5 March 2019

\section{Key words:}

Dengue; distributed lag non-linear model; El Niño; India; relative risk; temperature

Author for correspondence:

Srinivasa Rao Mutheneni, E-mail: msrinivas@ iict.res.in

\author{
Satya Ganesh Kakarla ${ }^{1}$, Cyril Caminade ${ }^{2,3}$, Srinivasa Rao Mutheneni ${ }^{1}$, Andrew \\ P Morse ${ }^{2,4}$, Suryanaryana Murty Upadhyayula ${ }^{5}$, Madhusudhan Rao Kadiri ${ }^{1}$ \\ and Sriram Kumaraswamy ${ }^{1}$
}

\begin{abstract}
${ }^{1}$ Applied Biology Division, CSIR-Indian Institute of Chemical Technology, Tarnaka, Hyderabad-500 007, Telangana, India; ${ }^{2}$ NIHR Health Protection Research Unit in Emerging and Zoonotic Infections, Liverpool L69 3GL, UK; ${ }^{3}$ Department of Epidemiology and Population Health, Institute of Infection and Global Health, University of Liverpool, Liverpool L35RF, UK; ${ }^{4}$ Department of Geography and Planning, School of Environmental Sciences, University of Liverpool, Liverpool L69 7ZT, UK and ${ }^{5}$ National Institute of Pharmaceutical Education and Research, Guwahati-781 032, Assam, India
\end{abstract}

\begin{abstract}
Dengue is a widespread vector-borne disease believed to affect between 100 and 390 million people every year. The interaction between vector, host and pathogen is influenced by various climatic factors and the relationship between dengue and climatic conditions has been poorly explored in India. This study explores the relationship between El Niño Southern Oscillation (ENSO), the Indian Ocean Dipole (IOD) and dengue cases in India. Additionally, distributed lag non-linear model was used to assess the delayed effects of climatic factors on dengue cases. The weekly dengue cases reported by the Integrated Disease Surveillance Program (IDSP) over India during the period 2010-2017 were analysed. The study shows that dengue cases usually follow a seasonal pattern, with most cases reported in August and September. Both temperature and rainfall were positively associated with the number of dengue cases. The precipitation shows the higher transmission risk of dengue was observed between 8 and 15 weeks of lag. The highest relative risk (RR) of dengue was observed at $60 \mathrm{~mm}$ rainfall with a 12 -week lag period when compared with 40 and $80 \mathrm{~mm}$ rainfall. The $\mathrm{RR}$ of dengue tends to increase with increasing mean temperature above $24^{\circ} \mathrm{C}$. The largest transmission risk of dengue was observed at $30^{\circ} \mathrm{C}$ with a $0-3$ weeks of lag. Similarly, the transmission risk increases more than twofold when the minimum temperature reaches $26^{\circ} \mathrm{C}$ with a 2 -week lag period. The dengue cases and El Niño were positively correlated with a 3-6 months lag period. The significant correlation observed between the IOD and dengue cases was shown for a 0-2 months lag period.
\end{abstract}

\section{Introduction}

Dengue is one of the most important mosquito-borne viral diseases in tropical and subtropical countries. It is transmitted through the bite of Aedes mosquitoes infected with dengue virus (DENV 1-4 serotypes). Aedes aegypti and Aedes albopictus are believed to be the main vectors of dengue virus in India $[1,2]$. Dengue causes a wide range of clinical symptoms including asymptomatic cases, acute febrile syndrome, severe and fatal cases of haemorrhagic manifestation that result in a significant fluid loss, which ultimately leads to shock. During the past five decades, the incidence of dengue has increased 30 -fold and it has become a major public health problem globally [3]. In 2012, WHO estimated that 50-100 million new dengue infections were occurring annually. Before 1970, only nine countries had experienced severe dengue epidemics. The disease is now endemic in more than hundred countries across the world. Over $50 \%$ of the world's population, mostly in the tropics, is identified at risk of dengue infection [4].

Dengue is highly endemic in Southeast Asia and the Western Pacific regions [5]. In recent years, the number of dengue cases also sharply increased in Southern, Northern and Central Americas [6]. Cases across the Americas, Southeast Asia and the Western Pacific have exceeded 1.2 million in 2008, 2.2 million in 2010 and over 3.2 million in 2015 [7]. In Southeast Asia, the disease has been one of the major causes of hospitalisation amongst children since the 1990s [8]. Asian countries contribute around $70 \%$ of global dengue burden. India alone contributes about $34 \%$ global dengue burden and two-thirds of India's population are estimated to be at risk of dengue infection [5].

Since the mid-1990s, dengue epidemics in India have become more frequent in urban zones and they also spread to new geographic regions [9]. This geographic expansion of dengue might be related to changes in eco-climatic factors, climate change, rapid urbanisation, rapid population growth, population movement and ineffective vector control operations 
[10]. The epidemiology of dengue in India was first described in 1780 and the first large-scale outbreak occurred in 1963 [11]. Later, subsequent outbreaks have been reported from different parts of India [2, 11]. India has reported all four serotypes (DENV 1, 2, 3 and 4) of dengue virus since 1956 for various parts of the country [12]. Since 2001, the total number of dengue cases has steadily increased in India. In the early 2000s, dengue was endemic in a few southern (Maharashtra, Karnataka, Tamil Nadu and Pondicherry), northern and northwestern states (Delhi, Rajasthan, Haryana, Punjab and Chandigarh). Recently, dengue expanded to many states including the Union Territories [9]. Not only has the number of cases and severity of disease also increased, dengue used to be restricted to urban areas, but it has now spread to rural areas [1].

Like most vector-borne diseases (VBD), the epidemiology of dengue consists of host (humans), vectors (Aedes mosquitoes) and pathogen (dengue virus). The VBD system is strongly influenced by climatic factors including temperature and rainfall. The global temperature has increased significantly over the $20^{\text {th }}$ century. By the end of the $21^{\text {st }}$ century, it has been predicted that the global mean temperature will rise between 1.1 and $6.4^{\circ} \mathrm{C}$ with respect to pre-industrial values [13]. Overall, these rising temperatures will enhance the transmission rate of mosquito-borne diseases and will allow the expansion of the vector into new geographic regions [14]. According to the Intergovernmental Panel on Climate Change (IPCC), approximately $1.5-3.5$ billion people worldwide will be at risk of dengue infection by 2080 due to climate change [13]. Temperature affects the life cycle of Aedes vectors including development and survival of the immature and mature stages, development and length of the gonotrophic cycle. Additionally, temperature also influences virus replication rates within the mosquitoes, their gonotrophic cycle and the adult size of mosquitoes [15]. High-temperature conditions tend to shorten the extrinsic incubation period within the mosquito vector and thus increase the odds of more mosquitoes becoming infectious during their life span. Rainfall provides vector breeding habitats, although the relationship between rainfall and dengue is non-linear; heavy rainfall can flush out breeding sites as well [16]. Relative humidity also favours the survival rate and biting activity of adult mosquito. This allows the infected female mosquitoes to complete more than one replication cycle of the virus [17].

The El Niño Southern Oscillation (ENSO) is a naturally occurring mode of tropical Pacific climate variability and it has a large impact on global and regional temperature and rainfall in the Tropics [18]. El Niño events are characterised by an increase in sea surface temperatures (SST) in the tropical eastern Pacific Ocean, while La Niña events are characterised by cooler than average SST in the same region. These events typically occur every 2-7 years and develop in association with large-scale oscillations in atmospheric pressure over the tropical Indian and Pacific oceans [19]. Studies have shown that there is an association between ENSO and the burden of mosquito-borne diseases in tropical and subtropical regions [20-22]. ENSO has a significant impact on the Indian monsoon and influences weather patterns worldwide [23]. El Niño events are associated with drier than average conditions in the Indian region [24]. Conversely, La Niña events are associated with increased rainfall in India. El Niño events have been positively associated with an increase in the number of malaria cases in Venezuela and India [25, 26]. However, a weak association was observed between ENSO events and dengue incidence in Bangladesh [27].

The Indian Ocean Dipole (IOD), also called the Indian Ocean Zonal Mode, was discovered in the late 1990s. The IOD is a natural climate mode of variability; it arises from ocean-atmosphere interaction, and is the largest mode of interannual climate variability in the tropical Indian Ocean [28, 29]. The IOD mode is characterised by the anomalous west-east SST gradient accompanying zonal wind anomalies over the equatorial Indian Ocean [28]. The positive phase of the IOD is associated with warmer than average SST in the western Indian Ocean; colder than average SST conditions in the southeast Indian Ocean, off of Sumatra; and anomalous easterlies appear around the central equatorial Indian Ocean [28]. During a positive IOD event, the East African region receives above normal rainfall, while rainfall is reduced in Indonesia and in Australia causing significant drought [30]. The IOD also plays an important role as a modulator of the Indian summer monsoon rainfall (ISMR) and influences the correlation between the ISMR and ENSO [31].

There are only a few studies that investigate the epidemiology of dengue virus and the impact of climate on dengue transmission across India $[16,32]$. Researchers have provided various predictions at global scale based on climate models. Hence, the current study aimed to estimate the effect of climate on dengue transmission in overdispersed datasets. A distributed lag non-linear model (DLNM) was used to examine the delayed lag effect of different climatic variables on dengue cases using data from 2010 to 2017 [33]. DLNM is a flexible model which simultaneously describes a nonlinear and delayed effect of different factors on disease burden of climate change on dengue incidence [34]. We utilised the DLNM method to investigate the lag effects of climate variables on dengue cases in India. This technique is based on the cross-basis function that examines a two-dimensional relationship along the dimensions of climate change (rainfall or temperature) and time lag, in weeks [33]. In addition, the best-fitting model with climate parameters will be used to develop a dengue forecast model for India. The results of the study may also provide useful information to allocate public health resources and mitigate the burden of dengue disease in India.

\section{Methods}

\section{Epidemiological data}

Weekly reports of dengue cases for India from 2010 to 2017 were collected and compiled by the Integrated Disease Surveillance Program (IDSP), Ministry of Health and Family Welfare, Government of India (http: \www.idsp.nic.in). Dengue cases are confirmed in the laboratory by the MAC ELISA method on the basis of the detection of IgM antibodies [32]. The institutional committee (CSIR - Indian Institute of Chemical Technology) approved the study and no patient samples were handled during the study. Hence, the consent from patients was waived as we dealt with the recorded data.

\section{Climate data}

The temperature data were derived using the NCEP-DOE 2 reanalysis dataset [35]. This reanalysis dataset is produced using a state-of-the-art analysis/forecast system to perform data assimilation using past observed climate data. The NCEP reanalysis data are a mix of climate model data, corrected by a large amount of climate observations (using assimilation techniques to produce a continuous gridded product in space and time as climate observations can be patchy). The model component of the reanalysis is consequently based on a climate model - some physical processes are resolved in the spectral grid space. This dataset is available on a T62 (spectral 
model grid truncation, it has pros and cons for solving physical processes in the earth system) global Gaussian grid (about $1.8^{\circ} \times 1.8^{\circ}$ ) from 1979 to present at daily time step. Rainfall was derived from the Tropical Rainfall Measuring Mission (TRMM) dataset. TRMM is available on a $0.25^{\circ} \times 0.25^{\circ}$ spatial grid covering the Tropics and sub-Tropics $\left(60^{\circ} \mathrm{N}-60^{\circ} \mathrm{S}\right)$ from 2010 to 2017 [36]. Time series of weekly rainfall, maximum, minimum and mean temperatures for India from 2010 to 2017 are shown in Fig. 1. Statistics summarizing the average weekly temperatures (maximum, minimum and mean temperatures) and rainfall are shown in Table 1.

The monthly El Niño-Southern Oscillation (ENSO) index used in this study is the Nino3.4 SST index (calculated for the region $170^{\circ} \mathrm{E}-120^{\circ} \mathrm{W}$ and $5^{\circ} \mathrm{N}-5^{\circ} \mathrm{S}$ ) available from the Climate Prediction Centre of the US National Weather Service (http:// www.cpc.ncep.noaa.gov/data/indices/sstoi.indices) [37]. Similarly, the IOD is represented by the SST difference between the western equatorial Indian Ocean $\left(50^{\circ} \mathrm{E}-70^{\circ} \mathrm{E}\right.$ and $\left.10^{\circ} \mathrm{S}-10^{\circ} \mathrm{N}\right)$ and the southeastern equatorial Indian Ocean $\left(90^{\circ} \mathrm{E}-110^{\circ} \mathrm{E}\right.$ and $10^{\circ} \mathrm{S}-0^{\circ}$ $\mathrm{N})$. This SST gradient is also known as the Dipole Mode Index (DMI). When the DMI is positive, the phenomenon is referred to as the positive phase of the IOD, and when it is negative, it is referred to as the negative phase of the IOD. The monthly DMI values were obtained from the Japan Agency for Marine Earth Science and Technology (http://www.jamstec.go.jp/frcgc/research/ d1/iod/DATA/dmi.monthly.txt) [28].

\section{Lag effects}

DLNM were separately fitted to investigate if there was a delayed impact of climatic factors on weekly dengue cases at country scale. The relationship between climatic factors and dengue cases was investigated by employing two distinct approaches. First, the association between dengue and climatic factors at different time lags (lag 0-25 weeks) was assessed using Pearson correlation analysis. We correlated the value of a particular climatic variable at week $i$ with the number of dengue cases by date of symptoms expressed at week $i+\tau$ where $\tau$ is the time lag (in weeks). The second part of the analysis was carried out using the DLNM package available in $\mathrm{R}$ software. We used DLNM combined with quasi Poisson regression analysis. The observed data of weekly dengue cases show overdispersion (e.g. the variance of dengue cases largely exceeds the mean), consequently quasi-Poisson regression was used to estimate the effects of independent variables (observed rainfall and temperature) on dependent variable (observed dengue cases). The median value of climatic parameters (Table 1) was defined as the baseline centring value for calculating relative risk (RR). The RR was based on the Poisson regression model adjusting for various confounders. $\mathrm{RR}$ is defined as 'the ratio of the probability of dengue occurring at a certain value of a climate variable to the probability of the event occurring at a reference value of the same climate variable'.

The DLNM framework has enough flexibility to represent the non-linear and delayed associations on lag scale between climatic factors and dengue cases based on cross basis function. Lag represents the time interval between exposure event and clinical outcome.

$$
Y_{t}=\text { quasiPoisson }\left(\mu_{t}\right), t=1,2,3, \ldots \ldots \ldots, n
$$

$$
\begin{aligned}
\log (\mu t)= & \alpha+\sum_{l=1}^{L} \beta 1(T t, l)+\sum_{l=1}^{L} \beta 2(R t, l)+s(\text { week }+\lambda) \\
& + \text { Year }+\varepsilon t
\end{aligned}
$$

where $t$ is the week of observation, $Y_{t}$ denotes the observed dengue counts in week $t, \log (\mu t)$ represents the logarithm of expected dengue cases in week $t, \alpha$ is the model intercept; $T_{t, l}$ and $R_{t, l}$ are the matrices obtained by applying the DLNM to temperature and rainfall; $\beta 1$ and $\beta 2$ are the coefficients of temperature and rainfall matrices, $l$ is the lag in months; $L$ is the maximum lag; $s($ week, $\lambda)$ is the natural cubic spline smoothing function of the calendar week. The model was adjusted by using a natural cubic spline for temperature with a maximum lag of 4-30 weeks, whereas base B-spline function was used for rainfall with a maximum lag of 25 weeks. We used the $\mathrm{R}$ software (version 3.3.3) with the 'dlnm' package to carry out our analysis [38].

Pearson cross-correlation coefficient test was used to investigate the lagged relationship between monthly dengue cases, ENSO and IOD data. The level of statistical significance was considered to be 0.05 . The correlation analysis was calculated by using the SYSTAT statistical software (version 13).

\section{Results}

The weekly dengue cases, temperature and rainfall from 2010 to 2017 are shown in Fig. 1. Between January 2010 and December 2017, a total of 174912 dengue cases were reported by IDSP in India. There was an average of about 420 weekly dengue cases and 21864 annual dengue cases. The highest number of cases (15 619) was reported during the 45th week of 2015 (e.g. first week of November). The weekly mean temperature and average rainfall were $23{ }^{\circ} \mathrm{C}$ and $23 \mathrm{~mm}$, respectively. Descriptive statistics of all dependent and independent variables are shown in Table 1. The majority of dengue cases $(72.47 \%)$ occurred from July to October during the monsoon period (Fig. 1). During this period, the median maximum and minimum temperatures were 28 and $21{ }^{\circ} \mathrm{C}$, respectively. It reveals that the weekly time-series dengue cases and climatic factors indicate a strong seasonal pattern. The seasonal peak of dengue cases varies from year to year, although most of the cases tend to occur during the Indian monsoon (Fig. 1). Dengue cases increased tremendously from 2012 to 2016; 2015 and 2016 were considered as major dengue epidemics in India. There is no synchronous relationship between weekly dengue cases and climatic factors but a delayed lag effect was detected.

The lagged relationship between weekly cumulative rainfall and dengue cases in three-dimensional pattern is shown in Fig. S1. It reveals a non-linear relationship between rainfall and dengue cases with delayed lag effect. The RR is minimal for low rainfall period and it gradually increases along with increases in rainfall. The peak risk $(\mathrm{RR}>2.5)$ was observed between 100 and $120 \mathrm{~mm}$ rainfall with 9-20 weeks lag, which decreased slowly during the following week. Figure 2 shows cross-sections of the twodimensional surface shown in Fig. S1 for fixed time lags at 3, 5, 10,15 and 20 weeks lag periods and for specific rainfall values, e.g. for $20,40,60,80,100$ and $120 \mathrm{~mm}$. The exposure-response analyses indicate that moderate dengue risk is observed between 40 and $60 \mathrm{~mm}$ rainfall with a 5-week lag period (Fig. 3). Rainfall ranging between 80 and $120 \mathrm{~mm}$ was associated with a higher RR and longer lag periods were observed (Fig. 3).

The association between mean temperature and dengue cases with a 30-week lag is shown in Fig. S2. The three-dimensional relationship between mean temperature and dengue cases shows a non-linear relationship and the RR increases with increasing temperature (Fig. S2). The largest RR (1.8) is shown between 28 and $30{ }^{\circ} \mathrm{C}$ with a $3-8$ weeks lag. Thereafter the risk gradually 

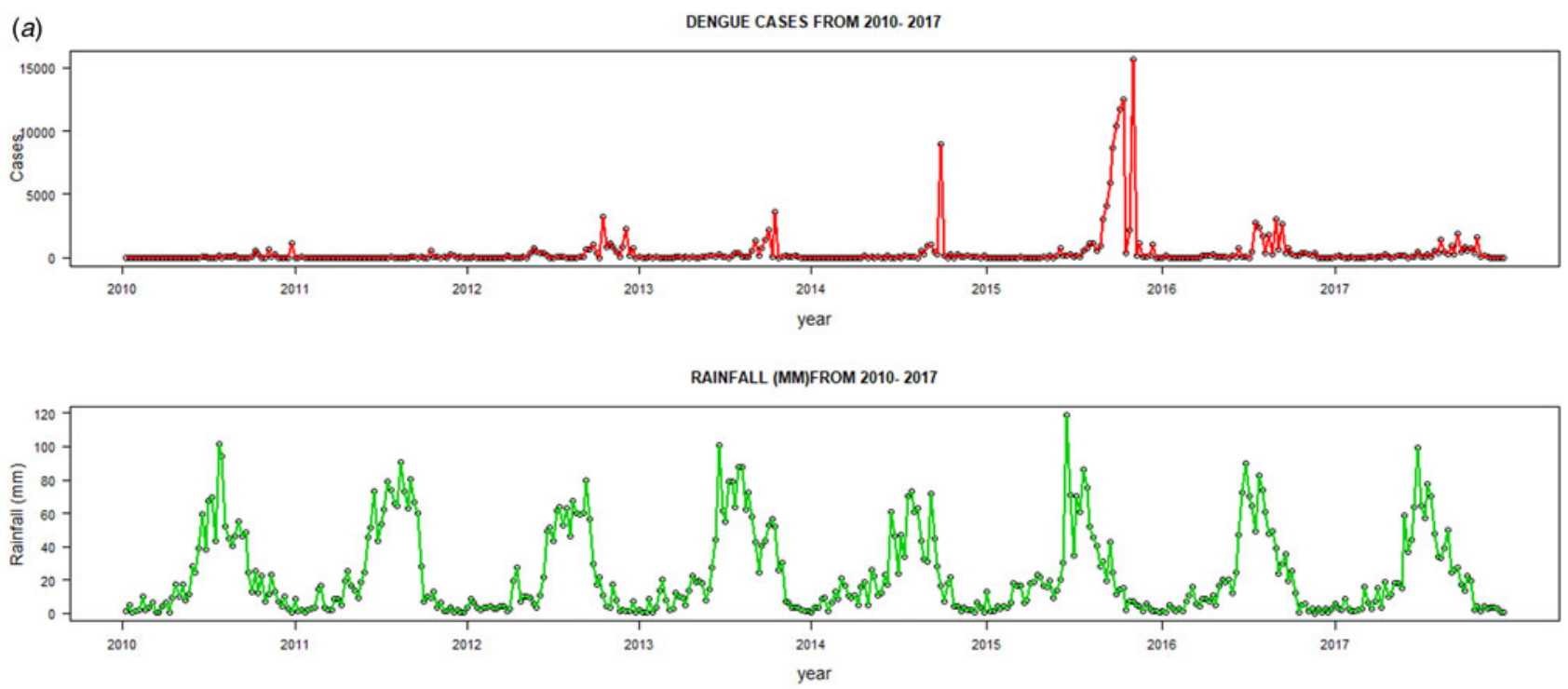

MAXIMUM,MEAN,MINIMUM TEMPERATURES FROM 2010- 2017

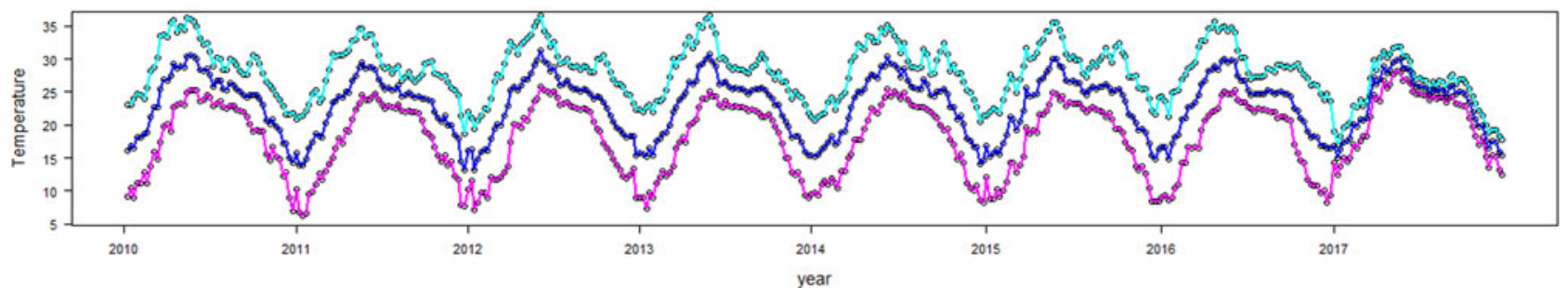

(b)

NINO3.4 standardized anomaly $2010-2017$

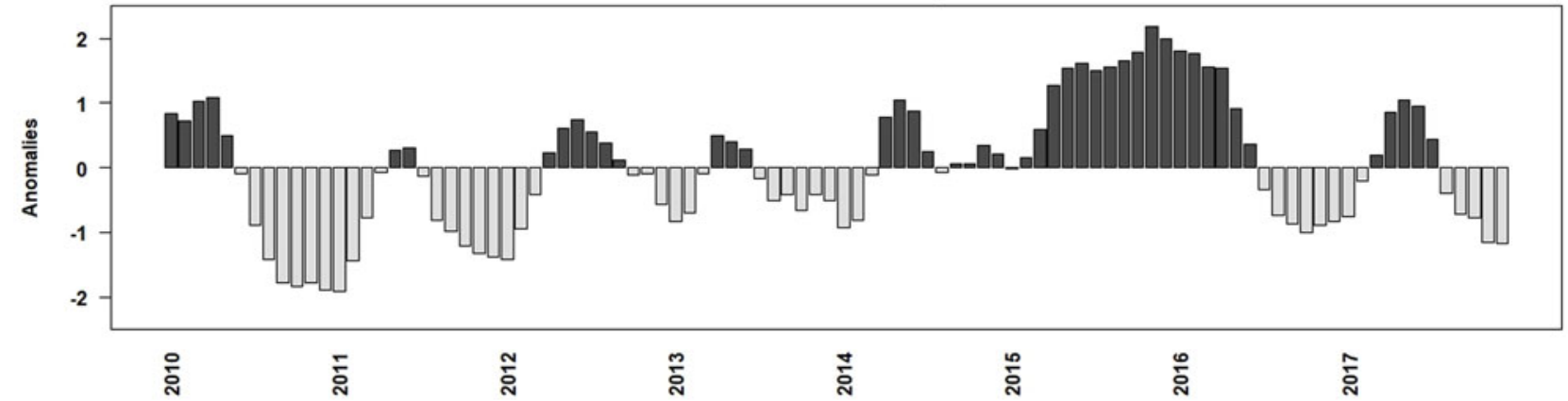

DMI standardized anomaly 2010-2017

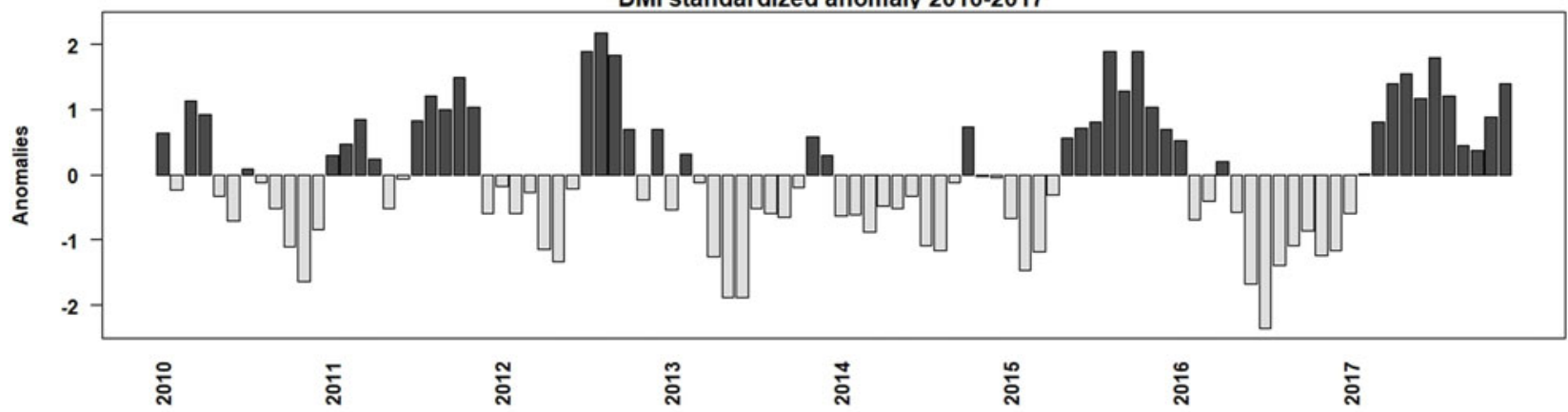

Fig. 1. Time-series plots of (a) weekly dengue cases, precipitation, maximum, minimum and mean temperature, (b) Nino3.4 and DMI indices during the period 2010-2017.

decreases with increasing temperature $\left(>30^{\circ} \mathrm{C}\right)$. A reversed $\mathrm{U}$-shaped lag responsive curve relationship was observed between dengue and mean temperature at different lag periods (Fig. 4).
The exposure-response analyses (Fig. 4) highlight that the RR by temperature at specific lags $(3,5,10,15$ and 20 weeks) and by lag at specific temperature $\left(24,26,28,30\right.$ and $\left.32{ }^{\circ} \mathrm{C}\right)$ was 
Table 1. Descriptive statistics of weekly information on weather and dengue cases from 2010 to 2017

\begin{tabular}{|c|c|c|c|c|c|c|c|}
\hline \multicolumn{8}{|c|}{ Descriptive statistics of weekly data } \\
\hline Variable & Mean & Minimum & Maximum & SD & \multicolumn{3}{|c|}{ Percentile } \\
\hline Cases & 419.5 & 0 & 15619 & 1475.54 & 10 & 56 & 211 \\
\hline Minimum temperature $\left({ }^{\circ} \mathrm{C}\right)$ & 18.18 & 6.25 & 28.11 & 5.49 & 13.15 & 19.41 & 22.80 \\
\hline Rainfall (in mm) & 23.22 & 0.11 & 119.09 & 25.01 & 3.65 & 12.98 & 38.82 \\
\hline
\end{tabular}

lag $=0$

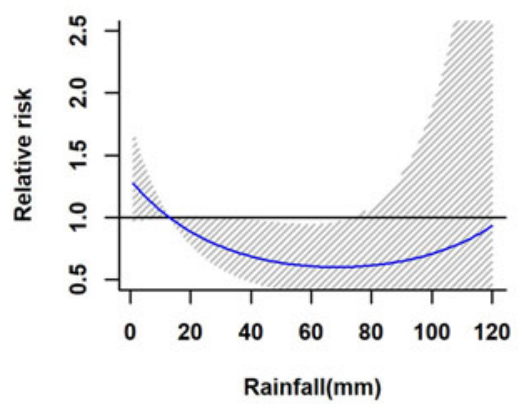

lag $=10$

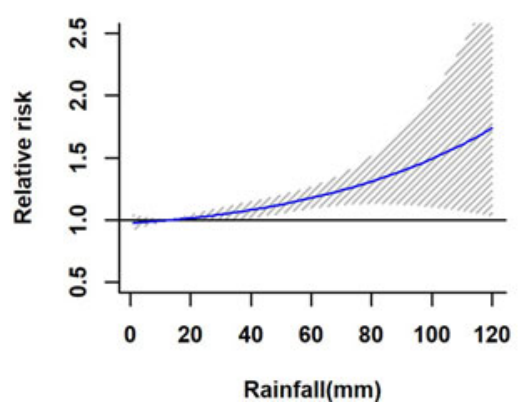

lag $=3$

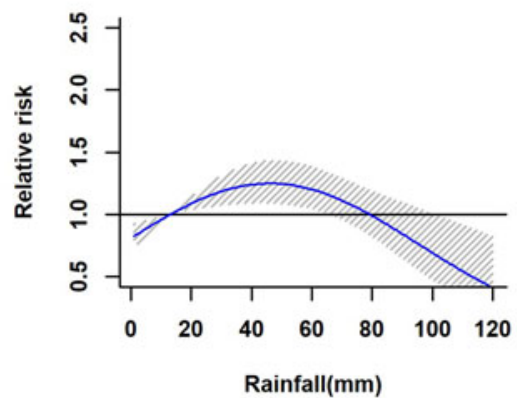

lag $=15$

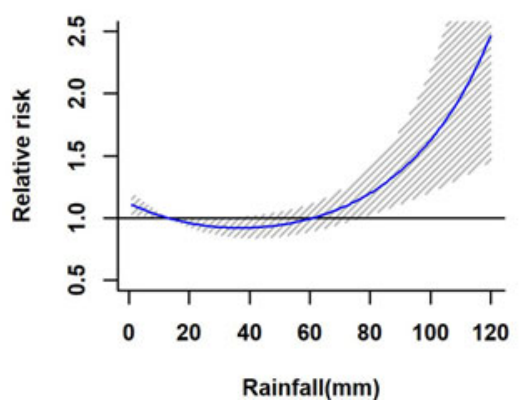

lag $=5$

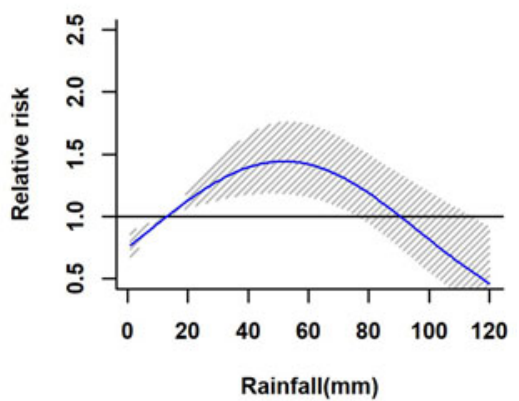

lag $=20$

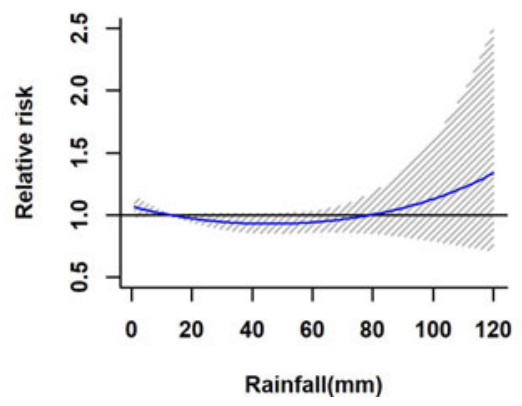

Fig. 2. The estimation of relative risk posed by rainfall at different time lags (in weeks). The solid blue line is the estimated non-linear curve; the shaded region indicates its $95 \%$ confidence interval.

observed. It was found that, the risk of disease transmission increases with increasing mean temperatures $(T)$ but high risk of disease transmission was further observed at optimum mean temperature range $\left(28-30^{\circ} \mathrm{C}\right)$ and the risk of disease transmission decreases for $T<24{ }^{\circ} \mathrm{C}$ and $T>32^{\circ} \mathrm{C}$ (Fig. 5). The associations between minimum and maximum temperature and dengue $\mathrm{RR}$ are presented as three-dimensional graphs in Fig. 6. The dengue $\mathrm{RR}$ is higher $(>1.2)$ when temperature leads $\mathrm{RR}$ by 1 week and when minimum temperature ranges between 22 and $25^{\circ} \mathrm{C}$. RR $>$ 1.4 is shown with 1 week lag when maximum temperature lies between 32 and $35^{\circ} \mathrm{C}$.

\section{Cross-correlation}

Temperature and rainfall exhibited significant correlations with dengue cases at different time lags (Table S1). The largest correlation $(r=0.32)$ is shown between rainfall and dengue at 12-week lag (Fig. 7). The positive correlation coefficient is observed between maximum temperature and dengue cases. Mean temperature also shows positive correlation with dengue cases at a 19-week lag period. The correlation between minimum temperature and dengue is significant but the correlation values are quite small.

\section{Effect of ENSO and IOD on dengue}

A weak synchronous correlation was observed between Nino3.4 $(r=0.3)$, DMI $(r=0.2)$ and dengue cases from 2010 to 2017. However, the largest El Niño events and the largest positive phases of the IOD coincide with the largest number of dengue cases reported in India in 2015 and 2016 (Fig. 1). Good correlation coefficients are shown between Nino3.4 $(r=0.5)$, 
Rainfall=20(mm)

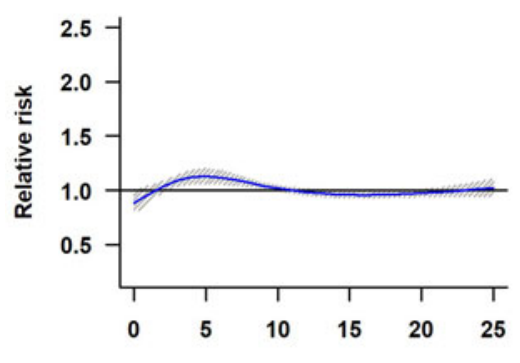

lag weeks

Rainfall=80(mm)

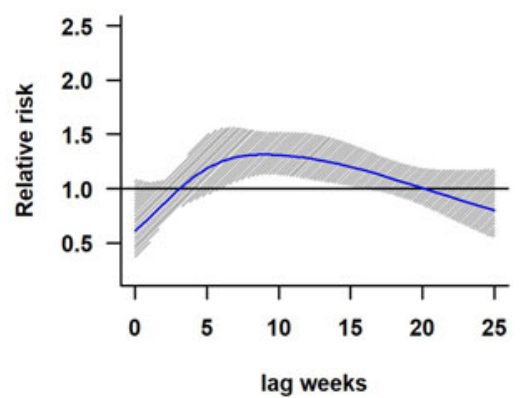

Rainfall $=40(\mathrm{~mm})$

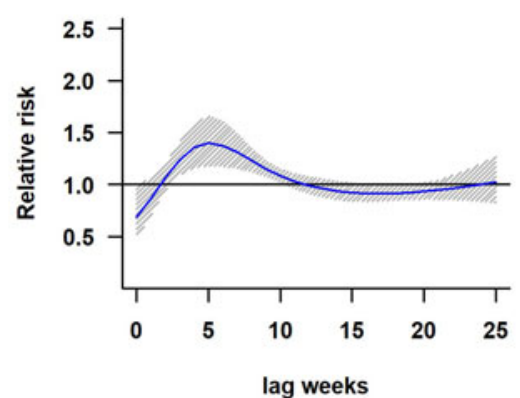

Rainfall $=100(\mathrm{~mm})$

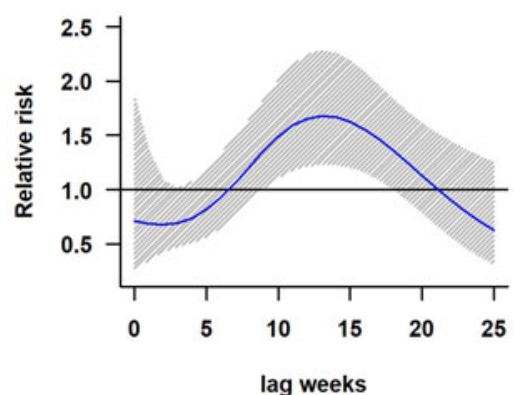

Rainfall $=60(\mathrm{~mm})$

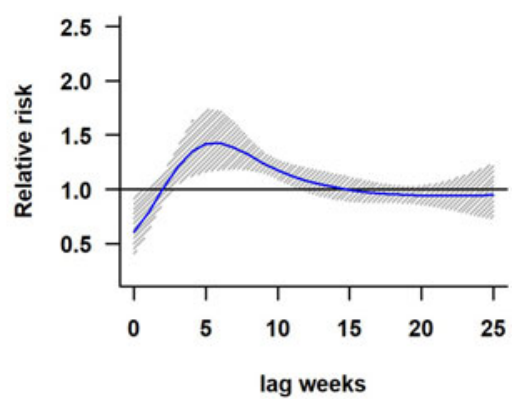

Rainfall=120(mm)

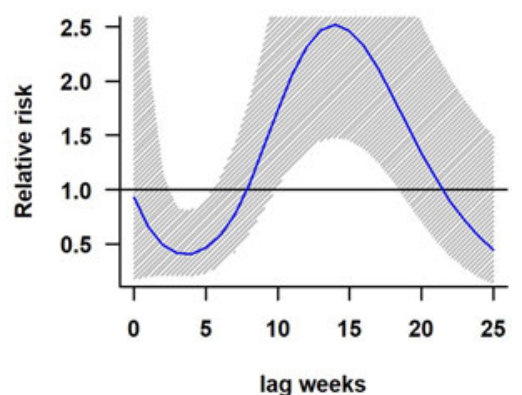

Fig. 3. The relative risk of dengue at different rainfall ranges. The solid blue line is the estimated non-linear curve; the shaded region indicates its $95 \%$ confidence interval.

DMI $(r=0.6)$ and dengue cases during strong El Niño period (in 2015), whereas negative correlation $(r=-0.6)$ between dengue cases and Nino3.4 and DMI indices are shown for 2016. Figure 8 depicts cross-correlations between Nino3.4 and DMI indices with dengue cases. Monthly Nino3.4 and DMI indices were significantly associated with the number of dengue cases at different time lags. Nino3.4 shows the largest significant correlation coefficient when the Nino3.4 index lead dengue cases by 3-6 months. Similarly, the highest significant correlation between the DMI index and dengue cases was found for a time lag ranging between 0 and 2 months (Table S2).

\section{Discussion}

Many studies have shown that temperature and rainfall are important drivers associated with the emergence of dengue. In the present study, a DLNM was utilised to understand the shortterm association between climate variables and dengue cases for different time lags. The results show that minimum temperature $\left(26^{\circ} \mathrm{C}\right)$, maximum temperature $\left(32^{\circ} \mathrm{C}\right)$ with $0-5$ weeks lag and rainfall $(60 \mathrm{~mm})$ with a lag of $8-12$ weeks are the most significant variables associated with an increase in the RR of dengue.

The non-linear relationship between rainfall and dengue is related to rainfall effects on the adult female mosquito lifecycle. Rainfall provides breeding habitats and opportunities for the proliferation of vectors in the environment. An increase in the amount of rainfall leads to more potential breeding sites, which, in turn, lead to an increase in the number of mosquitoes hatching. However, high rainfall may washout mosquito breeding sites thus having a negative effect on mosquito density [15]. This is consistent with our findings, the RR of dengue gradually increases as cumulative weekly rainfall increases from 40 to $60 \mathrm{~mm}$, then it decreases when rainfall exceeds $80 \mathrm{~mm}$. Similar results were observed in Brazil, namely that the risk of dengue infection increases during the rainy season when vector infestation reaches its peak [39]. Excepting rainfall intensity and magnitude, other man-made factors such as human activities, usage of water, storage patterns and drainage systems can create artificial breeding habitats for dengue vectors. In Southeast Asian countries, residents generally grow potted plants/flowers indoors and they tend to decorate roofs with hanging gardens. These man-made containers, if not emptied or cleaned frequently, can act as a breeding source for vectors well adapted to the urban environment. This can potentially further increase the risk of dengue transmission [40, 41]. In India, residents generally store water in different containers inside the house (especially in urban settings) and this water acts as a breeding source for Aedes vectors, thus further increasing the risk of dengue transmission [9]. In India, the south-west monsoon brings enough rainfall to sustain vector breeding sites $[42,43]$. Our findings show that the seasonality of the mosquito population is mainly driven by rainfall and ambient temperature conditions.

Temperature is one of the most important climatic factors, which influences the life cycle of the mosquito and pathogen development inside the vector. Temperature influences the length of gonotrophic cycle, larval development and growth rate of Aedes mosquitoes [44]. High temperatures are also associated with an increase in dengue incidence due to faster viral replication rates, shortened extrinsic incubation periods, increased blood-feeding behaviour, low mortality rates and high mosquito biting rates, leading to increased vectorial capacity. All these factors play a key role in disease transmission $[15,45]$. Previous studies have investigated the effect of temperature on the burden of dengue fever [46]; weekly minimum temperature was also strongly 


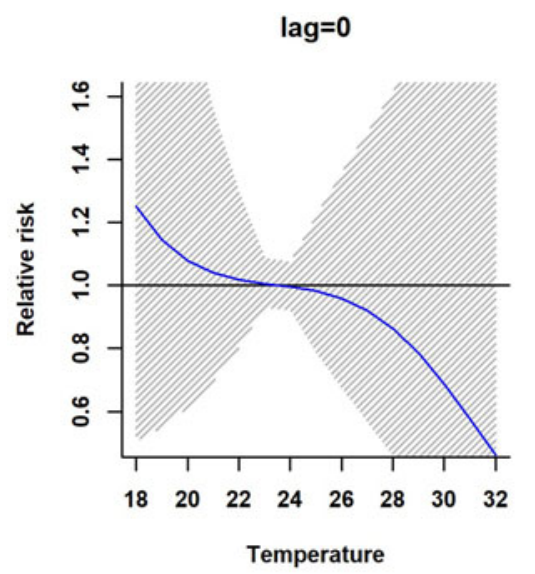

lag $=10$

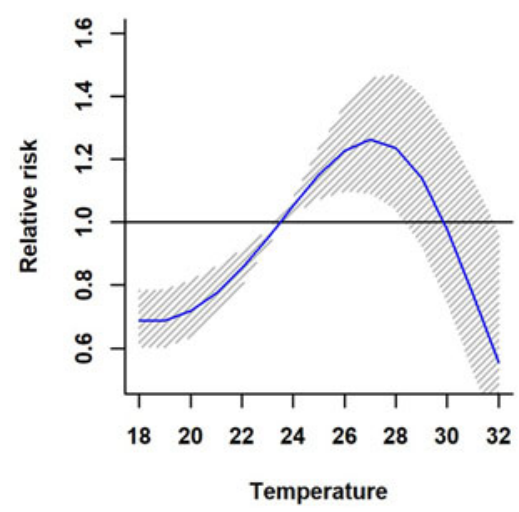

lag=3

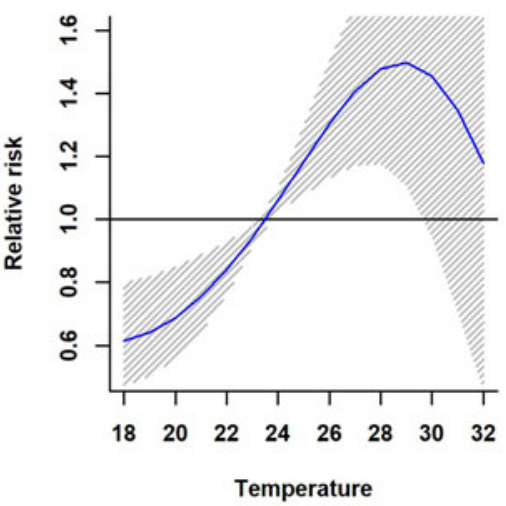

lag $=15$

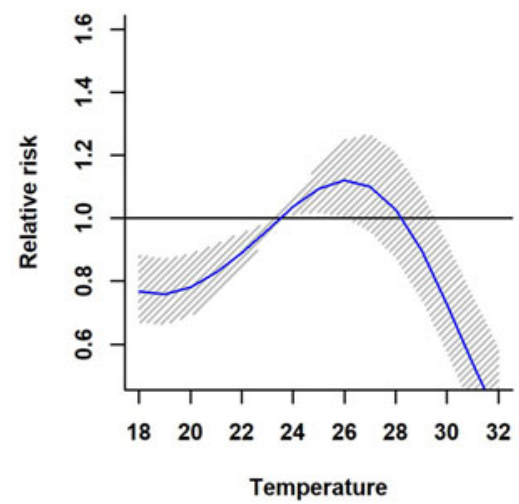

lag $=5$

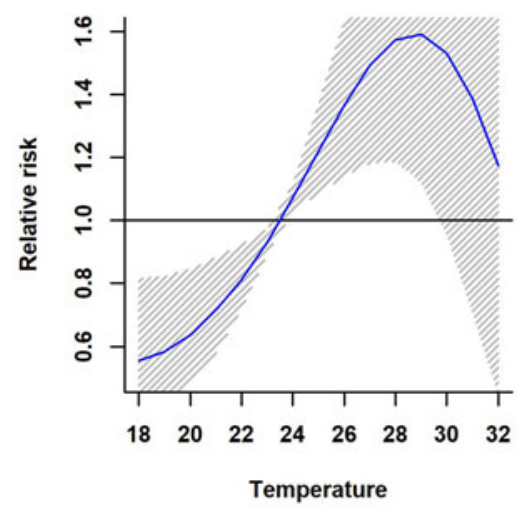

lag $=\mathbf{2 0}$

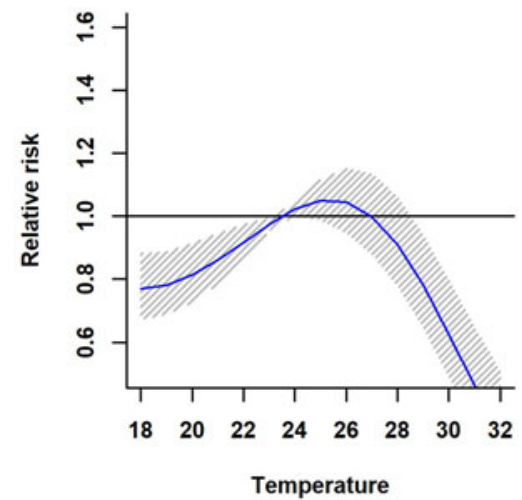

Fig. 4. Relative risk by mean temperature at specific lags. The solid red line is the estimated linear curve, with shaded region indicating its $95 \%$ confidence interval.

Temperature $=\mathbf{2 4}$

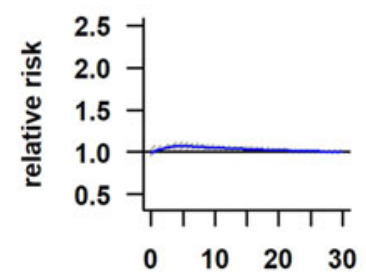

lag weeks

Temperature $=\mathbf{3 0}$

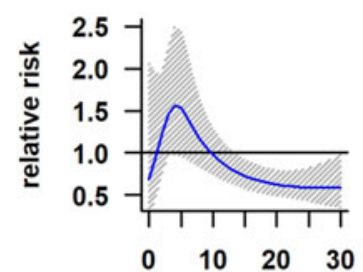

lag weeks
Temperature $=\mathbf{2 6}$

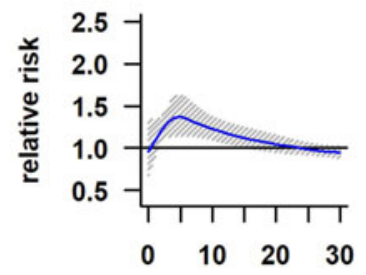

lag weeks

Temperature $=32$

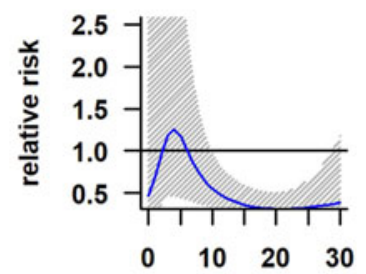

lag weeks

\section{Temperature $=\mathbf{2 8}$}

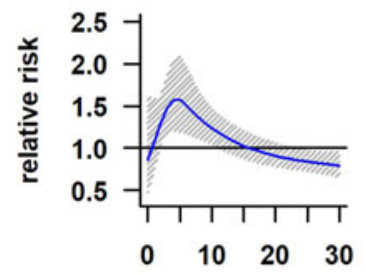

lag weeks
Fig. 5. Relative risk by lag at different mean temperatures. The solid red line is the estimated linear curve, with shaded region indicating its $95 \%$ confidence interval. 

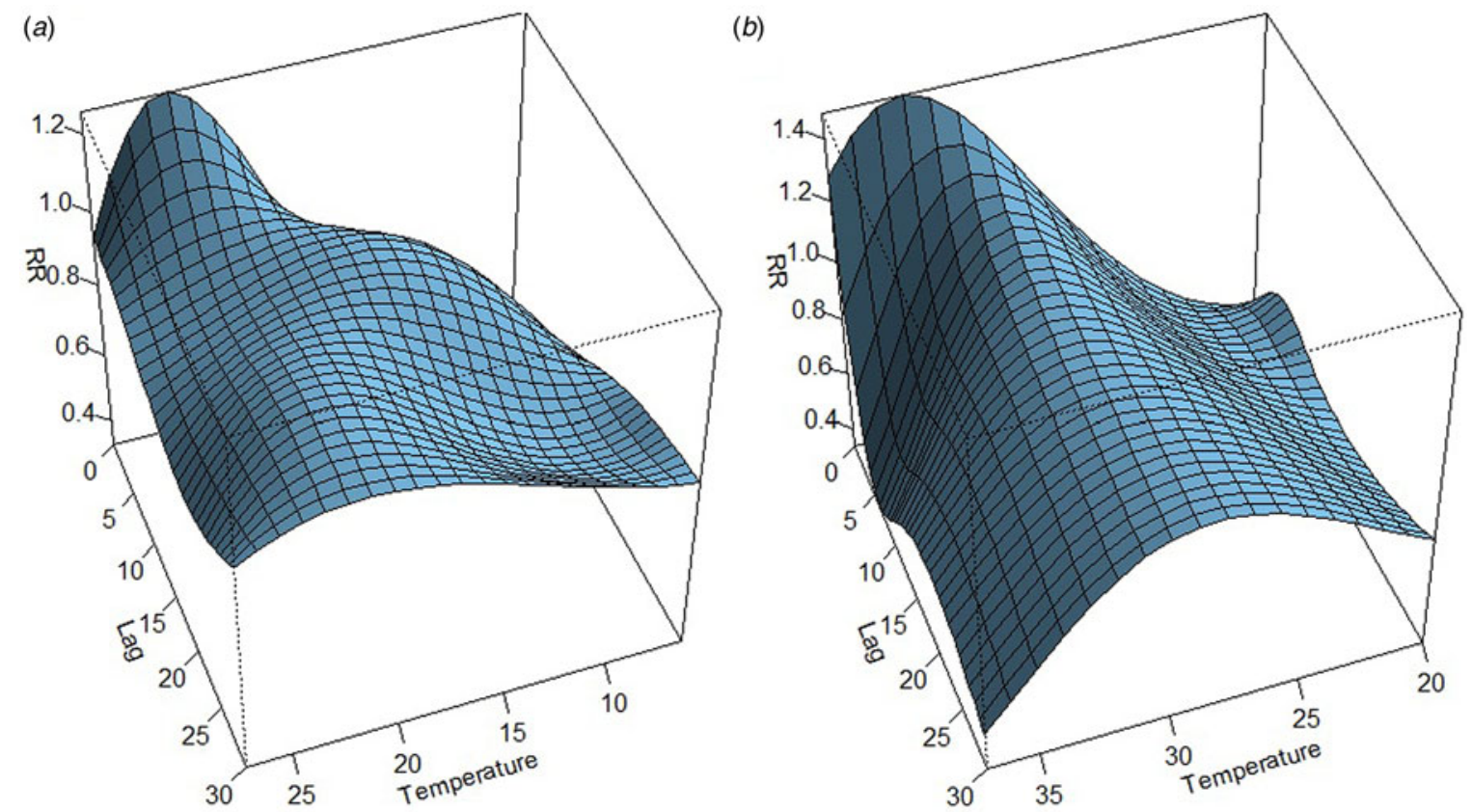

Fig. 6. The three-dimensional plot shows the association between weekly. (a) Minimum temperature. (b) Maximum temperature and relative risk of dengue at different lags.

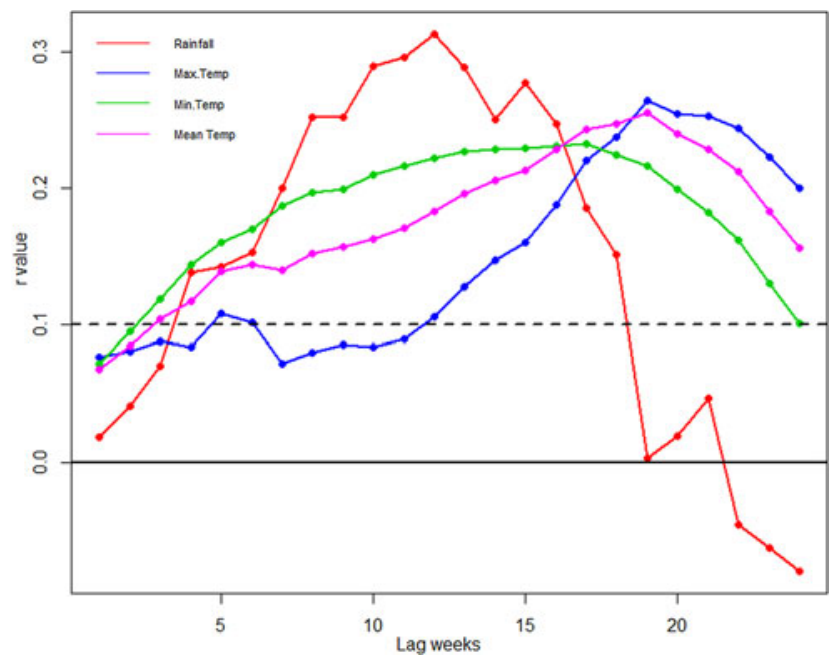

Fig. 7. Cross-correlation of dengue cases and climatic variable at $0-25$ weeks time lag. The dotted line stands for the significant correlation coefficients with $P<0.05$.

associated with vector development $[47,48]$. In this study, a positive association was observed between temperature and dengue cases for temperatures ranging between 24 and $30{ }^{\circ} \mathrm{C}$ at a lag of 0-4 weeks. Similar temperature ranges were also observed in China and Mexico [49, 50].

Studies found that water-borne disease and VBD outbreaks coincide with ENSO and IOD events [51, 52]. The present study found a weak synchronous correlation between ENSO $(r=0.3)$, IOD $(r=0.21)$ and dengue cases. In 2015-2016, a large number of dengue cases were observed during one of the largest El Niño events, a large positive phase of the IOD along with high rainfall. El Niño events are associated with a warming signal over the whole Tropics, warming temperature brought changes in the atmospheric circulation over the Indian Ocean which favours large rainfall during the monsoon period [31]. A significant positive relationship was observed between ENSO and monthly dengue incidence in Pacific Island nations [53]. Similarly, countries like Thailand, Mexico and Bangladesh have shown a positive association between ENSO, IOD and dengue cases [27, 54, 55].

The lagged effect of ENSO and IOD on the risk of dengue fever transmission is very important. The observed lag effects are biologically plausible, and they are consistent with former findings [52, 56, 57]. A study compiling all historical dengue outbreaks in India showed that most outbreaks in India are highly seasonal (occurring during the monsoon period) [9]. This study suggests that rainfall occurring at the end of the dry season enhances the risk of epidemics during the following monsoon season. Conversely, during pronounced drought conditions, water containers help to maintain the Aedes vector population around man-made water reservoirs in households. As a consequence, the pathogen remains during the dry season, and this can lead to an epidemic when the wet season returns. Non-climatic factors such as socio-ecological changes, viral serotypes, immunological factors, mosquito control approaches and population movement are important drivers for the spatial and temporal dynamics of dengue fever transmission [58, 59].

Earlier researchers found that dengue and its vectors are adapted to an urban setting, but in India, it has also spread to rural regions $[1,60,61]$. In recent years, the number of dengue cases has increased dramatically in India; hence it is crucial to develop a seasonal forecasting model to predict dengue prevalence for the next season. Our study has provided some basic information on temperature and rainfall threshold levels which might help to build a dengue early warning system to inform decisionmaking activities such as when to initiate preventive measures to reduce dengue mortality and morbidity.

This study has some limitations. First, the study duration (2010-2017) and sample size is relatively small. Our study highlights the granular association between climate and dengue at 


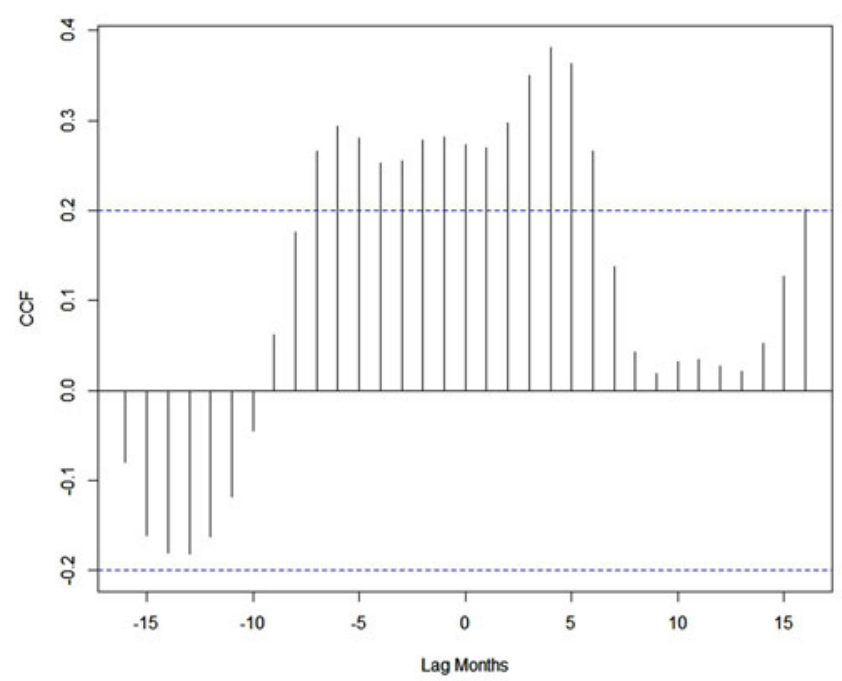

Fig. 8. Cross-correlation between NIÑO3.4, DMI indices and dengue cases.

country scale. Similar modelling exercise should be carried out at city or state level for effective management and control of the disease. As India has different climatic zones, future studies should focus on the development of forecasting models by climatic zone. Other important parameters, including socioeconomic and demographic factors such as population density, migration, vector density, virus serotype and immunity of the population should be included in future risk assessment studies to further understand this complex and fast-growing disease.

\section{Conclusion}

In conclusion, our findings revealed a non-linear relationship between climatic factors and dengue burden in India. The study shows that dengue is temperature-dependent and with increasing temperature the dengue cases increase above $24^{\circ} \mathrm{C}$. The estimated lagged effects are in accordance with the time required for the development of the Aedes vectors, for the extrinsic and intrinsic incubation periods of virus as well as the onset of clinical symptoms of dengue. The study also provides information for better understanding the effect of climate variables on dengue and can adapt a policy for control and preventive measures well in advance.

Supplementary material. The supplementary material for this article can be found at https://doi.org/10.1017/S0950268819000608

Author ORCIDs. (D) Srinivasa Rao Mutheneni, 0000-0003-3263-3905

Acknowledgements. The authors Srinivasa Rao Mutheneni and Sriram Kumaraswamy, Madhusudhan Rao Kadiri and Satya Ganesh Kakarla are grateful to the Director of the Council of Scientific and Industrial Research-Indian Institute of Chemical Technology, Hyderabad for his encouragement and support. Srinivasa Rao Mutheneni acknowledges the Ministry of Environment, Forest \& Climate Change, Government of India for funding the project ENVIS (Resource Partner on Climate Change and Public Health). Suryanarayana Murty Upadhyayula acknowledges support from the Ministry of Chemicals and Fertilizers, Govt. of India. The research was partly funded by the National Institute for Health Research Health Protection Research Unit (NIHR HPRU) in Emerging and Zoonotic Infections at the University of Liverpool in partnership with Public Health England (PHE) and Liverpool School of Tropical Medicine (LSTM). The views expressed are

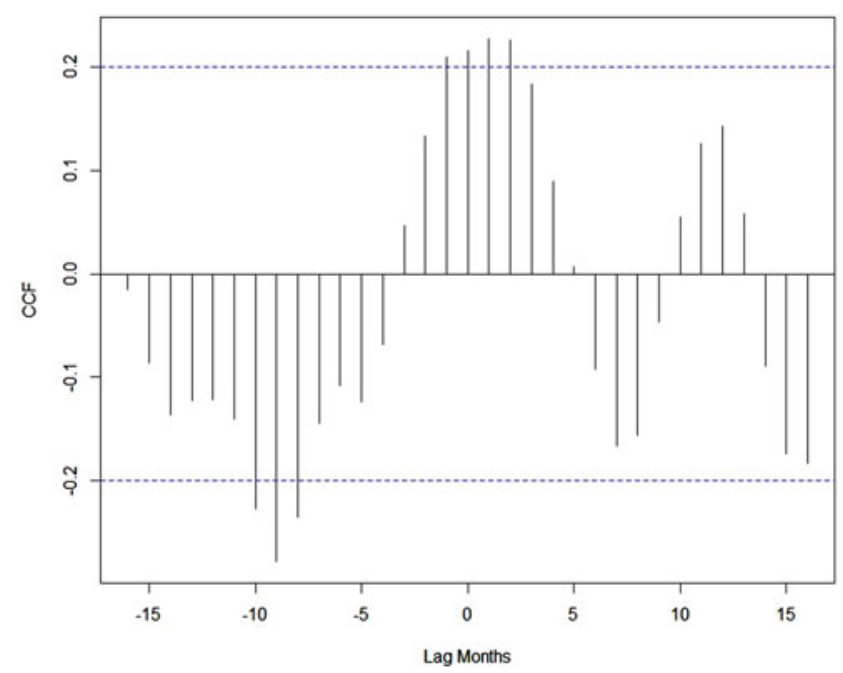

those of the authors and not necessarily those of the NHS, the NIHR, the Department of Health or Public Health England.

Conflict of interest. None.

\section{References}

1. Arunachalam N et al. (2004) Studies on dengue in rural areas of Kurnool District, Andhra Pradesh, India. Journal of American Mosquito Control Association 20, 87-90.

2. Chaturvedi UC and Nagar R (2008) Dengue and dengue haemorrhagic fever: Indian perspective. Journal of Bioscience 33, 429-441.

3. World Health Organisation (WHO) (2012) Global Strategy for dengue prevention and control, 2012-2020. Available at http://www.who.int/den guecontrol/9789241504034/en/ (Accessed 04 April 2017).

4. Brady OJ et al. (2012) Refining the global spatial limits of dengue virus transmission by evidence-based consensus. PLoS Neglected Tropical Diseases 6, e1760.

5. Bhatt S et al. (2013) The global distribution and burden of dengue. Nature 496, 504-507.

6. Gyawali N, Bradbury RS and Taylor-Robinson AW (2016) The epidemiology of dengue infection: harnessing past experience and current knowledge to support implementation of future control strategies. Journal of Vector Borne Diseases 53, 293-304.

7. World Health Organisation (WHO) (2017) Dengue and severe dengue. Available at http://www.who.int/mediacentre/factsheets/fs117/en/ (Accessed 06 April 2017).

8. World Health Organisation (1986) Dengue hemorrhagic fever, diagnosis, treatment and control. World Health Organization, Geneva, Switzerland.

9. Chakravarti A, Arora R and Luxemburger C (2012) Fifty years of dengue in India. Transactions of the Royal Society of Tropical Medicine and Hygiene 106, 273-282.

10. Gubler DJ (1998) Dengue and dengue hemorrhagic fever. Clinical Microbiology Review 11, 480-496.

11. Ramakrishnan SP et al. (1964) The epidemic of acute haemorrhagic fever, Calcutta, 1963; epidemiological inquiry. Indian Journal of Medical Research 52, 633-650.

12. Pandya G (1982) Prevalence of dengue infections in India. Defense Science Journal 4, 359-370.

13. IPCC. IPCC Fourth Assessment Report: Climate Change 2007. Available at http://www.ipcc.ch/publications_and_data.

14. Campbell-Lendrum D et al. (2015) Climate change and vector-borne diseases: what are the implications for public health research and policy? 
Philosophical Transactions of the Royal Society B: Biological Sciences 370, 20130552.

15. Morin CW, Comrie AC and Ernst K (2013) Climate and dengue transmission: evidence and implications. Environmental Health Perspectives 121, 1264-1272.

16. Jeelani S and Sabesan S (2013) Aedes vector population dynamics and occurrence of dengue fever in relation to climate variables in Puducherry, South India. International Journal of Current Microbiology and Applied Sciences 2, 313-322.

17. Donalisio MR and Glasser CM (2002) Vigilancia entomologica e controle de vectores do dengue. Revista Brasileira de Epidemiologia 5, 259-272.

18. Nicholls N (1993) El Niño-southern oscillation and vector-borne disease. Lancet 342, 1284e5.

19. Stewart-Ibarra AM and Lowe $\mathbf{R}$ (2013) Climate and no-climate drives of dengue epidemics in southern coastal Equator. American Journal of Tropical Medicine and Hygiene 88, 971-981.

20. Cazales B et al. (2005) Nonstationary influence of El Niño on the synchronous dengue epidemics in Thailand. PLoS Medicine 2, 313-318.

21. Chadee DD et al. (2007) Climate, mosquito indices and epidemiology of dengue fever in Trinidad (2002-2006). Annals of Tropical Medicine and Parasitology 101, 69-77.

22. Chretien J et al. (2015) Global climate anomalies and potential infectious disease risks: 2014-2015. PLOS Currents Outbreaks. 2015 Jan 26. Edition 1. doi: 10.1371/currents.outbreaks.95fbc4a8fb4695e049baabfc2fc8289f.

23. Kovats RS et al. (2003) El Niño and health. Lancet 362, 1481-1489.

24. Kumar KK et al. (2006) Unraveling the mystery of Indian monsoon failure during El Niño. Science 314, 115-119.

25. Delgado-Petrocelli L et al. (2012) Analysis of the El Niño/ LaNiña-southern oscillation variability and malaria in the EstadoSucre, Venezuela. Geospatial Health 6, S51-S57.

26. Dhiman RC and Sarkar S (2017) El Niño Southern Oscillation as an early warning tool for malaria outbreaks in India. Malaria Journal 16, 122.

27. Banu S et al. (2015) Impacts of El Niño Southern Oscillation and Indian Ocean Dipole on dengue incidence in Bangladesh. Scientific Reports 5, 16105.

28. Saji NH et al. (1999) A dipole mode in the tropical Indian Ocean. Nature 401, 360-363.

29. Webster PJ et al. (1999) Coupled ocean-atmosphere dynamics in the Indian Ocean during 1997-98. Nature 401, 356-360.

30. Hashizume M, Terao T and Minakawa N (2009) The Indian Ocean Dipole and malaria risk in the highlands of Western Kenya. Proceedings of the National Academy of Sciences 106, 1857-1862.

31. Ashok K et al. (2004) Individual and combined influences of ENSO and the Indian Ocean Dipole on the Indian summer monsoon. Journal of Climate 17, 3141-3155.

32. Mutheneni SR et al. (2017) Dengue burden in India: recent trends and importance of climatic parameters. Emerging Microbes and Infections 6, e70.

33. Talagala T (2015) Distributed lag nonlinear modelling approach to identify relationship between climatic factors and dengue incidence in Colombo District, Sri Lanka. Epidemiology Biostatistics and Public Health 12, e11522. doi: 10.2427/11522.

34. Gasparrini A, Armstrong B and Kenward MG (2010) Distributed lag non-linear models. Statistics in Medicine 29, 2224-2234.

35. Kanamitsu M et al. (2002) NCEP-DOE AMIP-II Reanalysis (R-2). Bulletin of American Meteorological Society 83, 1631-1643.

36. Huffman GJ et al. (2007) The TRMM multi-satellite precipitation analysis: quasi-global, multi-year, combined-sensor precipitation estimates at fine scale. Journal of Hydrometeorology 8, 38-55.

37. Climate Prediction Center (2018) Center for Weather and Climate Prediction, NOAA, USA (CPC-NOAA). http://www.cpc.ncep.noaa.gov/products/analy sis_monitoring/ensostuff/ensoyears.shtml (Accessed 30 September 2018).

38. R Core Team (2014) R: A language and environment for statistical computing. R Foundation for Statistical Computing, Vienna, Austria.
39. Aguiar M et al. (2015) Carnival or football, is there a real risk for acquiring dengue fever in Brazil during holidays seasons? Scientific Reports 5, 8462.

40. Wu PC et al. (2007) Weather as an effective predictor for occurrence of dengue fever in Taiwan. Acta Tropica 103, 50-57.

41. Sang S et al. (2015) Predicting unprecedented dengue outbreak using imported cases and climatic factors in Guangzhou, 2014. PLoS Neglected Tropical Diseases 9, e0003808.

42. Guhathakurta $\mathbf{P}$ et al. (2015) Observed changes in southwest monsoon rainfall over India during 1901-2011. International Journal of Climatology 135, 1881-1898.

43. Kolivras KN (2010) Changes in dengue risk potential in Hawaii, USA, due to climate variability and change. Climate Research Journal 42, 1-11.

44. Patz JA et al. (2005) Impact of regional climate change on human health. Nature 438, 310-317.

45. Watts DM et al. (1987) Effect of temperature on the vector efficiency of Aedes aegypti for dengue 2 virus. American Journal of Tropical Medicine and Hygiene 36, 143-152.

46. Tsai CT et al. (2012) Exploring the spatial and temporal relationships between mosquito population dynamics and dengue outbreaks based on climatic factors. Stochastic Environmental Research and Risk Assessment 26, 671-680.

47. Padmanabha $\mathbf{H}$ et al. (2012) An eco-physiological model of the impact of temperature on Aedes aegypti life history traits. Journal of Insect Physiology 58, 1597-1608.

48. Tun-Lin W, Burkot TR and Kay BH (2000) Effects of temperature and larval diet on development rates and survival of the dengue vector Aedes aegypti in North Queensland, Australia. Medical Veterinary Entomology 14, 31-37.

49. Xiang J et al. (2017) Association between dengue fever incidence and meteorological factors in Guangzhou, China, 2005-2014. Environmental Research 153, 17-26.

50. Colón-González FJ et al. (2013) The effects of weather and climate change on dengue. PLoS Neglected Tropical Diseases 7, e2503.

51. Banu S et al. (2014) Projecting the impact of climate change on dengue transmission in Dhaka, Bangladesh. Environment International 63, 137-142.

52. McMichael A et al. (1996) Climate Changes and Human Health. Geneva: WHO.

53. Hales S et al. (1999) El Niño and the dynamics of vector borne disease transmission. Environmental Health Perspective 107, 99-102.

54. Tipayamongkholgul $\mathbf{M}$ et al. (2009) Effects of the El Nino-Southern Oscillation on dengue epidemics in Thailand, 1996-2005. BMC Public Health 9, 422.

55. Hurtado-Diaz M et al. (2007) Short communication: impact of climate variability on the incidence of dengue in Mexico. Tropical Medicine and International Health 12, 1327-1337.

56. Arcari P, Tapper N and Pfueller S (2007) Regional variability in relationships between climate and dengue/DHF in Indonesia. Singapore Journal of Tropical Geography 28, 251-272.

57. Hii YL et al. (2012) Optimal lead time for dengue forecast. PLoS Neglected Tropical Diseases 6, e1848.

58. Gubler DJ (2011) Dengue urbanization and globalization: the unholy trint of $21^{\text {st }}$ century. Tropical Medicine and Health 39, 3-11.

59. Hay SI et al. (2009) Etiology of interepidemic periods of mosquito borne diseases. Proceedings of the National Academy of Sciences 97, 9335-9339.

60. Hiscox A et al. (2013) Risk factors for the presence of Aedes aegypti and Aedes albopictus in domestic water-holding containers in areas impacted by the Nam Theun 2 Hydroelectric Project, Laos. American Journal of Tropical Medicine and Hygiene 88, 1070-1078.

61. Tsuda Y et al. (2006) Different spatial distribution of Aedes aegypti and Aedes albopictus along an urban-rural gradient and the relating environmental factors examined in three villages in Northern Thailand. Journal of American Mosquito Control Association 22, 222-228. 